\title{
ELABORAÇÃO DE PROPOSTAS PARA ALTA SEGURA COM USO DO \\ DIAGNOSIS-RELATED GROUPS
}

\section{PREPARATION OF PROPOSALS FOR SECURE DISCHARGE WITH THE USE OF DIAGNOSIS-RELATED GROUPS}

\section{Aline Aparecida Campos Reis}

Médica com Título de Especialista em Nefrologia pela Sociedade Brasileira de Nefrologia. MBA em Gestão Empresarial pela Fundação Getúlio Vargas. Pós-graduação em DRG Sistemas de Saúde pela Faculdade de Ciências Médicas de Minas Gerais

\section{Marcelo Augusto Nascimento}

Administrador hospitalar, professor orientador. 


\section{RESUMO}

Introdução: Dentre as várias estratégias que podem ser utilizadas para aumentar a entrega de valor em saúde, encontram-se a redução de readmissões não planejadas e o planejamento de alta segura. A metodologia Diagnosis-related groups (DRG) permite a geração de relatórios que fornecem informações sobre características epidemiológicas da população atendida, uso de leito hospitalar, grau de complexidade assistencial e taxas de readmissão hospitalar. A análise desses relatórios possibilita a tomada de decisões com ênfase em qualidade assistencial e redução de desperdícios.

Objetivos: O objetivo geral do trabalho foi elaborar propostas de alta segura a partir da análise de relatórios gerados pela metodologia DRG de um hospital privado. Os objetivos específicos foram: análise de relatórios gerados pelo DRG, com determinação das características dos DRG's gerados, permanência média hospitalar e taxa de readmissão em 30 dias.

Método: Trata-se de um estudo observacional, do tipo transversal, com análise de relatórios epidemiológicos e de desempenho gerados pelo sistema DRG. Foram analisados todos os DRG's gerados a partir das altas codificadas entre 01/09/2019 e 31/09/2020.

Resultados: Foram codificadas 2.257 altas no período estudado, com 69,78\% dos DRG's gerados sendo cirúrgicos. O case mix global foi 1,3057, sendo o cirúrgico $(1,4433)$ mais complexo que o clínico $(0,9879)$. Apesar dos DRG's cirúrgicos serem mais frequentes, os DRG's clínicos consumiram 53,04\% do tempo de permanência. Houve ineficiência do uso do leito hospitalar e consumo de diárias acima do previsto, tanto em DRG's clínicos quanto cirúrgicos, com desperdício de 71,5 admissões clínicas e 228,7 cirúrgicas no período analisado. A expectativa era de uma permanência média realizada por DRG clínico e cirúrgico de 3,7 e 1,4 dias, respectivamente. No entanto, o que se observou foi uma permanência média realizada de 4,1 dias para internações clínicas e de 1,6 para cirúrgicas. A taxa de readmissão em 30 dias foi de 3,19\%, com DRG's clínicos e cirúrgicos apresentando proporção semelhante de reinternações.

Conclusão: O emprego da metodologia DRG permite a elaboração de protocolos de alta segura para auxílio da entrega de valor. Propõe-se para o hospital em estudo, as seguintes estratégias de alta segura: 
1. para internações clínicas e internações cirúrgicas de emergência: (A) planejamento de alta; (B) educação e preparação de paciente e cuidadores e (C) definição da forma de seguimento pós-alta (via telefone ou através de agendamento de consulta ambulatorial conforme o risco de readmissão em 30 dias);

2. para cirurgias eletivas: elaboração de protocolos de enhanced recovery after surgery (ERAS) para as principais cirurgias eletivas realizadas no hospital.

Palavras-chave: Tempo de permanência; Readmissão; Custos; Saúde baseada em valor.

\section{ABSTRACT}

Introduction: Among the various strategies that can be used to increase the delivery of health value, there are the reduction of unplanned readmissions and the planning of safe discharge. The Diagnosis-related groups (DRG) methodology allows the generation of reports that provide information on the epidemiological characteristics of the population served, use of hospital beds, degree of care complexity and rates of hospital readmission. The analysis of these reports enables decision making with an emphasis on quality of care and reduction of waste.

Objectives: The general objective of the work was to elaborate safe discharge proposals based on the analysis of reports generated by the DRG methodology of a private hospital. The specific objectives were: analysis of reports generated by the DRG, with determination of the characteristics of the generated DRG's, average hospital stay and readmission rate in 30 days.

Method: This is an observational, cross-sectional study, with analysis of epidemiological and performance reports generated by the DRG system. All DRG's generated from the codified highs between 09/01/2019 and 09/31/2020 were analyzed.

Results: 2,257 discharges were coded in the studied period, with $69.78 \%$ of the generated DRGs being surgical. The global case mix was 1.3057, with the surgical (1.4433) being more complex than the clinical (0.9879). Although surgical DRG's are more frequent, clinical DRG's consumed $53.04 \%$ of the length of stay. There was inefficiency in the use of the hospital bed and consumption of daily rates above expectations, both in clinical and surgical DRGs, with a loss of 71.5 clinical admissions and 228.7 surgical ones in the analyzed period. The expectation was an average stay performed by clinical and surgical DRG of 3.7 and 1.4 days, respectively. 
However, what was observed was an average stay of 4.1 days for clinical admissions and 1.6 days for surgery. The 30-day readmission rate was 3.19\%, with clinical and surgical DRGs showing a similar proportion of readmissions.

Conclusion: The use of the DRG methodology allows the elaboration of high security protocols to aid the delivery of value. The following hospital discharge strategies are proposed for the hospital under study:

1. for clinical admissions and emergency surgical admissions: (A) discharge planning; (B) education and preparation of patients and caregivers and (C) definition of the form of postdischarge follow-up (by telephone or by scheduling an outpatient appointment according to the risk of readmission in 30 days);

2. for elective surgeries: elaboration of enhanced recovery after surgery (ERAS) protocols for the main elective surgeries performed at the hospital.

Keywords: Length of stay; Readmission; Costs; Health-based value. 


\section{INTRODUÇÃO}

Para qualquer organização, seja qual for o setor envolvido, a melhoria de desempenho está associada ao estabelecimento de objetivos que unam interesses e atividades de todos os stakeholders. Particularmente no setor de saúde, os stakeholders possuem vários interesses distintos e não raro conflitantes, tais como: acesso a serviços, lucro, qualidade, contenção de custos, segurança, conveniência, atenção centrada no paciente e satisfação. A falta de clareza de objetivos leva a abordagens divergentes, conflitos e lentificação do processo de melhoria de performance (PORTER, 2010).

Para Poter e Teisberg (2006), a meta principal a ser coletivamente buscada e atingida é a entrega de elevado valor em saúde, com valor definido como a relação entre os resultados assistenciais e seus custos. Esse objetivo é o que realmente interessa ao paciente, além de permitir a união de interesses de todos os demais atores envolvidos no sistema de saúde.

Dentre as várias estratégias que podem ser utilizadas para aumentar a entrega de valor, encontram-se a redução de readmissões não planejadas e o planejamento de alta segura. Conforme Dias (2015), readmissões desnecessárias indicam elementos disfuncionais no sistema de saúde, acarretam riscos indevidos aos pacientes e custos desnecessários ao sistema. Dessa forma, o estudo das readmissões hospitalares permite monitorar resultados e tomada de decisões gerenciais que auxiliam na alocação de recurso, garantia de acesso e oferta adequada de serviços.

Barnes et al. (2016) enfatizam que o planejamento de alta com cuidado específico por paciente hospitalizado possibilita a efetividade do leito operacional, a redução de eventos adversos, a segurança e a experiência do paciente, possibilitando uma maior gestão dos recursos, dentre eles o custo com mão de obra, equipamentos e leitos.

Existem várias ferramentas gerenciais que podem ser utilizadas para garantir desempenho e redução de custos, dentre elas o Diagnosis-related groups (DRG). A metodologia DRG foi inicialmente desenvolvida na Universidade de Yale, Estados Unidos, na década de 1960. Os pesquisadores, liderados por Robert B. Fetter e John Thompson, desenvolveram pesquisas nas áreas de gerência, planejamento e revisão de utilização hospitalar (NORONHA et al, 1991).

Segundo Rimler et. al. (2015), os objetivos principais da elaboração do sistema DRG foram: (1) estimular médicos a utilizar recursos hospitalares de forma mais racional e econômica; (2) documentar a relação entre decisões médicas e administrativas e (3) definir produtos e serviços hospitalares por diagnósticos. Com base nesses objetivos, para cada 
paciente admitido em ambiente hospitalar é atribuído um código DRG (considerado um produto assistencial). A codificação pelo sistema DRG difere, entretanto, da Classificação Internacional de Doenças (CID). Cada doença possui a sua classificação pela CID, ao passo que um grupo assistencial DRG pode englobar vários códigos da CID.

Além da codificação, a ferramenta DRG permite a geração de relatórios que fornecem, dentre outras, informações sobre características epidemiológicas da população atendida, uso de leito hospitalar, grau de complexidade assistencial e taxas de readmissão hospitalar. A análise desses relatórios fornece, portanto, subsídios para tomadas de decisão com ênfase em qualidade assistencial e consumo racional de recursos (CALDERON et al., 2019).

As instituições de saúde que priorizam qualidade assistencial e entrega de valor ao paciente, mas sem perder sustentabilidade e competitividade, devem lançar mão de ferramentas, tais como a metodologia DRG. Conhecer a população atendida, os desfechos assistenciais e seus custos podem permitir o estabelecimento de estratégias de governança, como a elaboração de protocolos de alta segura. Diante disso, questiona-se se quais propostas de alta segura, com foco em qualidade assistencial e consumo racional de recursos, podem ser elaboradas a partir da análise dos relatórios gerados pela metodologia DRG em um hospital privado.

\section{OBJETIVOS}

Geral: Elaborar propostas de alta segura para um hospital privado a partir da análise de relatórios gerados pela plataforma DRG.

Específicos: Analisar os relatórios gerados pelo sistema DRG do hospital; determinar características dos DRG's gerados, permanência média hospitalar e taxa de readmissão em 30 dias.

\section{REFERENCIAL TEÓRICO}

$\mathrm{Na}$ atualidade, apesar de todo avanço tecnológico e seu consequente elevado consumo de recursos médicos e hospitalares, os pacientes nem sempre percebem esse avanço como entrega de valor e manifestam isso como insatisfação com profissionais e provedores de saúde. Dessa forma, existe um movimento global para redirecionar o posicionamento estratégico dos sistemas de saúde (PORTER; TEISBERG, 2006). 
Os resultados desejados para alguns pacientes não necessariamente se aplicam a outros com o mesmo episódio de doença. Assim, valor em saúde implica em atenção centrada no paciente e não nas doenças (PORTER; LEE, 2013).

Para avaliar entrega de valor em saúde é fundamental a definição e mensuração de indicadores de resultado, muito embora isso não seja uma tarefa simples. Entregar valor não significa número de procedimentos, mas a relação entre os desfechos assistências e seus custos durante todo o ciclo do tratamento do paciente. Mesmo os indicadores considerados de resultados são, muitas vezes, de processos. Para melhor mensuração de resultados, Porter (2010) sugeriu uma hierarquia de desfechos clínicos, dividindo-a em três camadas: (1) relacionada ao estado de saúde atingido após tratamento (exemplos: sobrevida, mortalidade, capacidade funcional); (2) referente ao cuidado e processo de recuperação (exemplos: ocorrência de eventos adversos, complicações e tempo para reabilitação) e (3) relacionada à manutenção da saúde (exemplos: tempo livre de recorrência, complicações associadas ao tratamento de longo prazo, manutenção da independência para tarefas diárias).

Na busca de entrega de valor, várias ferramentas de gestão podem ser utilizadas para mensurar desfechos e custos, dentre elas a metodologia DRG. Essa metodologia enfatiza a atenção centrada no paciente, com objetivos de garantir qualidade assistencial, reduzir desperdícios e, consequentemente, otimizar consumo de recursos (CORTES et al., 2016).

O sistema DRG é considerado a maior revolução em modelos de gestão hospitalar, uma vez que a sua adoção pelo Medicare, programa governamental norte-americano de seguro de saúde, evitou o colapso do sistema no país. Por sua simplicidade de uso, transparência para as partes envolvidas, baixo custo de operação, capacidade de controlar o desperdício e induzir a entrega de valor, tal sistema passou a ser utilizado em todo o mundo. Atualmente, é empregado na maioria ou totalidade dos países da América do Norte, Ásia, Europa e Oceania (SANTOS et al., 2018).

A disseminação e sucesso mundial da aplicação dessa ferramenta pode ser justificada pelo aumento exponencial dos custos com saúde, o gera uma grande preocupação com desperdícios e contenção de despesas. Nessa perspectiva, para aumento de viabilidade financeira, várias instituições e sistemas de saúde, tanto em países de alta renda quanto em países de baixa e média renda, têm abandonado os modelos de remuneração por serviços ( $f e e$ for-service) e adotado o pagamento baseado no bundle DRG. A expectativa é a redução de gastos hospitalares, uma vez que o modelofee-for-service estimula o consumo de serviços para 
aumento de receitas, ao passo que o modelo de pagamento baseado no DRG permite uma remuneração pré-fixada, prospectiva, de acordo com a sua codificação (MENG et al., 2020).

Para Couto et al. (2018), a experiência de sete anos com a implantação software DRG possibilitou a deteç̧ão de quatro oportunidades de melhoria da qualidade e redução dos desperdícios em saúde, sendo elas: (1) utilização adequada do leito hospitalar conforme as necessidades reais do paciente; (2) segurança assistencial com a redução dos eventos adversos; (3) redução das internações hospitalares desnecessárias, que poderiam ser identificadas e tratadas na atenção primária, como exemplo as internações sensíveis à atenção primária e (4) redução das readmissões em 30 dias não planejadas.

Um dos focos do presente estudo e da metodologia DRG, as readmissões não planejadas são definidas como as readmissões hospitalares dos pacientes ocorridas em até 30 dias por complicações relacionadas à internação anterior, independente se a internação anterior ocorreu no mesmo hospital ou não (BERRY et al., 2018). Segundo Barnes et al. (2016), o planejamento do cuidado específico por paciente hospitalizado direciona a alta segura, possibilitando a continuidade do cuidado e redução das readmissões não planejadas, o que reduz desperdícios, melhora a qualidade assistencial, a segurança e a sustentabilidade.

Como medidas para garantir a alta segura, várias intervenções vêm sendo propostas e estudadas, tais como: planejamento de alta, seguimento por telefone, agendamento de consultas pós-alta, educação de pacientes com incentivo a autocuidado, treinamento de acompanhantes e visitas domiciliares. Leppin et al. (2014), por exemplo, em metanálise com 42 estudos randomizados e controlados, observaram que várias dessas intervenções podem reduzir readmissões precoces, sobretudo as relacionadas a apoiar e incentivar o autocuidado do paciente no período de transição entre o hospital e o domicílio.

Especificamente nas internações hospitalares para cirurgias eletivas, como medida de "cuidado continuado" e preparação para alta segura, cresce a utilização dos protolocos de Otimização da Recuperação Pós-operatória (enhanced recovery after surgery - ERAS em inglês), também conhecidos como fast-track surgery. A adoção desses protocolos objetiva redução de custos, mas sem impacto na qualidade de assistência entregue ao paciente (KEHLET et al., 2008).

O conceito de ERAS foi inicialmente desenvolvido na Dinamarca na década 1990 para cirurgias colorretais e encontra-se, desde então, em contínuo desenvolvimento para esta e várias outras modalidades cirúrgicas. Os protocolos de ERAS objetivam aumentar a segurança assistencial e a satisfação de pacientes em sistemas hospitalares através da utilização de pacotes 
de cuidados clínicos que fornecem abordagens consistentes ao cuidado perioperatório. São, assim, empregadas abordagens multidisciplinares, colaborativas e baseadas em evidências científicas para cuidados perioperatórios com intuito de melhorar a atenção pré, intra e pósoperatória. (BLUMENTHAL, 2019).

Segundo Lau et al. (2016), os protocolos de ERAS fornecem aos pacientes planos transformativos para: (1) minimizar a dor; (2) reduzir a administração de opióides; (3) retornar a função gastrointestinal; (4) reduzir as complicações infecciosas e respiratórias no pósoperatório e (5) diminuir o tempo de internação hospitalar. Em suma, esses protocolos quando conjunta e harmonicamente empregados visam o retorno da rotina normal do paciente o mais rápido possível, sem perder o foco na entrega de valor ao paciente.

\section{METODOLOGIA}

Trata-se de estudo observacional, do tipo transversal, com análise de relatórios epidemiológicos e clínicos gerados pelo sistema DRG em um hospital geral que atende pacientes de uma operadora de saúde suplementar, no interior do estado de Minas Gerais, Brasil.

Dentre os estudos observacionais, caracterizados pela não interferência do pesquisador, os estudos transversais analisam a situação de exposição e efeito de uma população em um único momento.

As principais vantagens dos estudos transversais são a simplicidade e agilidade de execução, além de serem relativamente econômicos e permitirem avaliar a prevalência associada aos agentes suspeitos e a descrição da população em análise. Como desvantagens desse tipo de estudo, destacam-se: não quantificação de risco de desenvolver a doença ou o evento em análise; não aparecimento da sequência temporal do fenômeno em estudo; impossibilidade de associação causa-efeito e risco de indução de associações ou associações falsas ou fortuitas.

O hospital, sede do presente estudo, é uma unidade assistencial de internação, que não possui pronto atendimento na mesma estrutura. Seu edifício possui $4.000 \mathrm{~m}^{2}$ distribuídos em cinco andares, com 16 apartamentos, 28 enfermarias e 10 leitos de UTI. O Centro Cirúrgico é formado por seis salas cirúrgicas, sendo uma de pequenos procedimentos e as demais de médio e grande porte. A instituição atende a todos requisitos regulatórios e, atualmente, organiza e estrutura seus processos para obtenção de certificações e acreditações hospitalares. 
O pronto-atendimento encontra-se em edifício distinto ao hospital. Pacientes com indicação de internação são transportados por ambulância até o hospital.

As internações são eletivas (para cirurgias programadas) ou em caráter de urgência (com pacientes clínicos e cirúrgicos encaminhados do pronto-atendimento da operadora de saúde).

\section{a. Obtenção da amostra}

O estudo avaliou as 2.257 altas codificadas, do hospital em questão, ocorridas entre 01/09/2019 a 31/09/2020.

\section{b. $\quad$ Coleta de dados}

Foram usados dados secundários do banco de dados do DRG do hospital.

A ferramenta DRG registra valores de variáveis como idade, CID principal, CIDs secundários, associados ao consumo de insumos hospitalares por comorbidades, gênero do paciente, data de internação, entre outros, gerando categorias padronizadas de diagnósticos. A versão deste software é a v34.0 do Medicare Severity-Diagnosis Related Group (MS-DRG), que utiliza o CID-10 como base.

O sistema gera relatórios de desempenho a partir dos dados codificados.

Não é possível o acesso ao nome do paciente, com proteção de sua identidade.

\section{c. $\quad$ Análise e apresentação dos resultados}

Os dados serão apresentados de forma descritiva e ilustrados em tabelas.

\section{d. $\quad$ Considerações éticas}

Houve liberação do projeto pela Diretoria Administrativa e pelo Comitê de Ética do referido hospital.

\section{RESULTADOS}

Foram analisadas 2.257 altas hospitalares, codificadas entre 01/09/2019 e 31/09/2020. A maior parte dos casos foram cirúrgicos $(69,78 \%)$. O case mix global encontrado foi 1,3057, sendo o case mix cirúrgico $(1,4433)$ mais elevado que o clínico $(0,9879)$. TABELA 1

Tabela 1 - Dados gerais das altas codificadas

\begin{tabular}{|l|l|}
\hline Registros saídas & 2.257 \\
\hline Média saídas/mês & 173,62 \\
\hline DRG's cirúrgicos & \multicolumn{2}{|c|}{1.575} \\
\hline DRG's clínicos & \multicolumn{2}{|c|}{682} \\
\hline Case mix global & 1,3057 \\
\hline
\end{tabular}




\begin{tabular}{|l|l|}
\hline Case mix clínico & 0,9879 \\
\hline Case mix cirúrgico & 1,4433 \\
\hline
\end{tabular}

prevalentes representaram 44,84\% das altas codificadas, sendo em sua grande maioria DRG's cirúrgicos. TABELA 2.

Tabela 2 - DRG’s (cirúrgicos e clínicos) mais prevalentes entre as altas codificadas

\begin{tabular}{|l|l|l|l|}
\hline DRG & Descrição & Quantidade & Percentual \\
\hline 419 & $\begin{array}{l}\text { Colecistectomia laparoscópica sem exploração do } \\
\text { ducto comum sem CC/MCC }\end{array}$ & 146 & $6,47 \%$ \\
\hline 134 & $\begin{array}{l}\text { Outras cirurgias do ouvido, nariz, boca e garganta sem } \\
\text { CC/MC }\end{array}$ & 130 & $5,76 \%$ \\
\hline 743 & $\begin{array}{l}\text { Cirurgia uterina e dos anexos para doenças não } \\
\text { neoplásicas sem CC/MCC }\end{array}$ & 126 & $5,58 \%$ \\
\hline 263 & Ligadura e remoção de veia & 120 & $5,32 \%$ \\
\hline 951 & Outros fatores que influenciam o estado de saúde de de & 113 & $5,01 \%$ \\
\hline 489 & $\begin{array}{l}\text { Cirurgia do joelho sem diagnóstico principal de } \\
\text { infecção sem CC/MC }\end{array}$ & $5,83 \%$ \\
\hline 670 & Cirurgia transuretral sem CC/MCC & 44 & $2,16 \%$ \\
\hline 352 & Cirurgias de Hérnia Inguinal e Femoral sem CC/MCC & 60 & $2,66 \%$ \\
\hline 690 & Infecções do rim e trato urinário sem MCC & 59 & $2,44 \%$ \\
\hline 502 & $\begin{array}{l}\text { Apendicectomia sem diagnóstico principal complexo } \\
\text { sem CC/MCC }\end{array}$ & 55 & $44,84 \%$ \\
\hline & Representatividade no banco de dados & 1.012 & \\
\hline
\end{tabular}

Os 10 DRG's cirúrgicos mais frequentes representaram $41,47 \%$ das altas codificadas, sendo os mais prevalentes o 419 (Colecistectomia laparoscópica sem exploração do ducto comum sem CC/MCC) e o 134 (Outras cirurgias do ouvido, nariz, boca e garganta, sem CC/MC). Dentre os DRG's clínicos, o mais prevalente foi o 951 (outros fatores que influenciam o estado de saúde), com percentual de 5,01\%, seguido pelo 690 (infecções do rim e trato urinário sem MCC) com 2,61\% de prevalência. TABELAS 3 e 4.

Tabela 3 - DRGs cirúrgicos mais prevalentes entre as altas codificadas

\begin{tabular}{|l|l|l|l|}
\hline DRG & Descrição & Quantidade & Percentual \\
\hline 419 & $\begin{array}{l}\text { Colecistectomia laparoscópica sem exploração do } \\
\text { ducto comum sem CC/MCC }\end{array}$ & $6,47 \%$ \\
\hline 134 & $\begin{array}{l}\text { Outras cirurgias do ouvido, nariz, boca e garganta sem } \\
\text { CC/MC }\end{array}$ & $5,76 \%$ \\
\hline 743 & $\begin{array}{l}\text { Cirurgia uterina e dos anexos para doenças não } \\
\text { neoplásicas sem CC/MCC }\end{array}$ & 126 & $5,58 \%$ \\
\hline 263 & Ligadura e remoção de veia 120 & $5,32 \%$ \\
\hline
\end{tabular}




\begin{tabular}{|l|l|l|l|}
\hline 489 & $\begin{array}{l}\text { Cirurgia do joelho sem diagnóstico principal de } \\
\text { infecção sem CC/MC }\end{array}$ & $4,83 \%$ \\
\hline 670 & Cirurgia transuretral sem CC/MCC & 94 & $4,16 \%$ \\
\hline 352 & Cirurgias de hérnia inguinal e femoral sem CC/MCC & 60 & $2,66 \%$ \\
\hline 343 & $\begin{array}{l}\text { Apendicectomia sem diagnóstico principal complexo } \\
\text { sem CC/MCC }\end{array}$ & 55 & $2,44 \%$ \\
\hline 502 & Cirurgias do tecido mole sem CC/MCC & 51 & $2,26 \%$ \\
\hline 355 & $\begin{array}{l}\text { Cirurgias de hérnia exceto inguinal e femoral sem } \\
\text { CC/MC }\end{array}$ & 45 & $1,99 \%$ \\
\hline & Representatividade no banco de dados & 936 & $41,47 \%$ \\
\hline
\end{tabular}

Tabela 4 - DRG's clínicos mais prevalentes entre as altas codificadas

\begin{tabular}{|l|l|l|l|}
\hline DRG & Descrição & Quantidade & Percentual \\
\hline 951 & Outros fatores que influenciam o estado de saúde & 113 & $5,01 \%$ \\
\hline 690 & Infecções do rim e trato urinário sem MCC & 59 & $2,61 \%$ \\
\hline 177 & Infecções respiratórias e inflamações com MCC & 54 & $2,39 \%$ \\
\hline 552 & Problemas clínicos do dorso sem MCC & 39 & $1,73 \%$ \\
\hline 558 & Tendinite, miosite e bursite sem MCC & 23 & $1,02 \%$ \\
\hline 866 & Doença viral sem MCC & 20 & $0,89 \%$ \\
\hline 392 & $\begin{array}{l}\text { Esofagite, gastroenterite e outras doenças digestivas } \\
\text { sem MCC }\end{array}$ & 19 & $0,84 \%$ \\
\hline 301 & Doenças vasculares periféricas sem CC/MCC & 16 & $0,71 \%$ \\
\hline 176 & Embolia pulmonar sem MCC & 15 & $0,66 \%$ \\
\hline 641 & $\begin{array}{l}\text { Doenças nutricionais e metabólicas diversas sem } \\
\text { MCC }\end{array}$ & 14 & $0,62 \%$ \\
\hline & Representatividade no banco de dados & 372 & $16,48 \%$ \\
\hline
\end{tabular}

Apesar dos DRG's cirúrgicos serem os mais frequentes, os DRG's clínicos representaram 53,04\% do tempo de permanência realizado no período estudado. A média de permanência hospitalar por DRG clínico foi de 4,1 dias, ao passo que a mesma média para DRG cirúrgico foi de 1,6 dias. Tanto em casos cirúrgicos quanto clínicos, houve permanência realizada acima da prevista pelos DRG's gerados. TABELA 5.

Tabela 5 - Relação entre permanência prevista x realizada (em dias) para DRG's clínicos e cirúrgicos

\begin{tabular}{|l|l|l|l|l|l|}
\hline Tipo de DRG & Quantidade & Previsto & Realizado & Diferença & $\begin{array}{l}\text { Permanência } \\
\text { média (PM)* }\end{array}$ \\
\hline Clínico & 682 & $\begin{array}{l}2.525,4 \\
(\mathrm{PM} \mathrm{3,7)}\end{array}$ & $2.789,7$ & 264,7 & 4,1 \\
\hline Cirúrgico & 1.575 & $\begin{array}{l}2.150,0 \\
(\mathrm{PM} 1,4)\end{array}$ & $2.470,2$ & 320,2 & 1,6 \\
\hline Total & 2.257 & $4.675,0$ & $5.259,9$ & 584,9 & 2,3 \\
\hline
\end{tabular}


Com a ineficiência do uso do leito hospitalar, devido ao consumo de diárias hospitalares acima do previsto, perderam-se 71,5 admissões clínicas e 228,7 admissões cirúrgicas. TABELA 6.

Tabela 6 -Ineficiência operacional do custo fixo (meta $\leq 100 \%)$ e média de admissões perdidas em DRG's clínicos e cirúrgicos

\begin{tabular}{|l|l|l|}
\hline Tipo DRG & Percentual de ineficiência & Média de admissões perdidas \\
\hline Clínico & $110,5 \%$ & 71,5 \\
\hline Cirúrgico & $114,9 \%$ & 228,7 \\
\hline
\end{tabular}

Apesar da maior proporção de DRG's cirúrgicos codificados, o número de óbitos foi maior em DRG's clínicos. TABELA 7.

Tabela 7 -Relação de óbitos com DRG's clínicos e cirúrgicos

\begin{tabular}{|l|l|l|}
\hline & Número de óbitos & Proporção de óbitos \\
\hline DRG's clínicos & 45 & $79,95 \%$ \\
\hline DRG's cirúrgicos & 12 & $21,05 \%$ \\
\hline Total altas codificadas & 57 & $100 \%$ \\
\hline
\end{tabular}

Os pacientes idosos (idade igual ou maior a 60 anos) representaram 31,37\% das altas codificadas, mas a proporção de óbitos por faixa etária foi expressivamente maior nesse grupo $(94,74 \%)$. A faixa etária de maior proporção de óbitos foi a maior ou igual a 80 anos (70,18\%), muito embora esse grupo represente apenas $11,87 \%$ da população analisada. TABELA 8.

Tabela 8 - Relação entre faixa etária e proporções de saídas e óbitos

\begin{tabular}{|l|l|l|l|l|}
\hline Faixa etária & Saídas & $\begin{array}{l}\text { Proporção } \\
\text { saídas }\end{array}$ & $\begin{array}{l}\text { Número de } \\
\text { óbitos }\end{array}$ & $\begin{array}{l}\text { Proporção } \\
\text { óbitos }\end{array}$ \\
\hline Recém-nascido (até 28 dias) & 0 & - & 0 & - \\
\hline Maior ou igual 29 dias a 17 anos & 84 & $3,72 \%$ & 0 & - \\
\hline 18 a 59 anos & 1.465 & $64,91 \%$ & 3 & $5,26 \%$ \\
\hline 60 a 69 anos & 246 & $10,90 \%$ & 4 & $7,02 \%$ \\
\hline 70 a 79 anos & 194 & $8,60 \%$ & 10 & $17,54 \%$ \\
\hline Maior ou igual a 80 anos & 268 & $11,87 \%$ & 40 & $70,18 \%$ \\
\hline Total & 2.257 & $100 \%$ & 57 & $100 \%$ \\
\hline
\end{tabular}

A taxa de readmissão em 30 dias foi de 3,19\%, com uma proporção semelhantes entre DRG's clínicos e cirúrgicos. TABELA 9 e 10.

Tabela 9 - Número de readmissões em 30 dias

\begin{tabular}{|l|l|}
\hline Tipo DRG & Número de readmissões \\
\hline
\end{tabular}




\begin{tabular}{|l|l|}
\hline Clínico & 44 \\
\hline Cirúrgico & 40 \\
\hline Total & 84 \\
\hline
\end{tabular}

Tabela 10 - DRG's com maior prevalência de readmissões em 30 dias

\begin{tabular}{|l|l|l|l|}
\hline DRG & Descrição & Quantidade & $\begin{array}{l}\% \text { em } \\
\text { saídas }\end{array}$ \\
\hline 670 & Cirurgias transuretral sem CC/MCC & 6 & $0,27 \%$ \\
\hline 134 & $\begin{array}{l}\text { Outras cirurgias do ouvido, nariz, boca e garganta sem } \\
\text { CC/MCC }\end{array}$ & 4 & $0,18 \%$ \\
\hline 552 & Problemas clínicos do dorso sem MCC & 4 & $0,18 \%$ \\
\hline 951 & Outros fatores que influenciam o estado de saúde & 4 & $0,18 \%$ \\
\hline 690 & Infecções do rim e trato urinário sem MCC & 4 & $0,18 \%$ \\
\hline 641 & Doenças nutricionais e metabólicas sem MCC & 3 & $0,13 \%$ \\
\hline 192 & Doença pulmonar obstrutiva crônica sem CC/MCC & 3 & $0,13 \%$ \\
\hline 343 & $\begin{array}{l}\text { Apendicectomia sem diagnóstico principal complexo sem } \\
\text { CC/MCC }\end{array}$ & 2 & $0,09 \%$ \\
\hline 392 & $\begin{array}{l}\text { Esofagite, gastroenterite e outras doenças digestivas sem } \\
\text { MCC }\end{array}$ & 2 & $0,09 \%$ \\
\hline 661 & Cirurgias do rim e ureter não-oncológicas sem CC/MCC & 2 & $0,09 \%$ \\
\hline 982 & $\begin{array}{l}\text { Cirurgia extensa não relacionada ao diagnóstico principal } \\
\text { com CC }\end{array}$ & 2 & $0,09 \%$ \\
\hline
\end{tabular}

\section{DISCUSSÃO}

No hospital estudado, houve ineficiência do uso do leito hospitalar e consumo de diárias acima do previsto, tanto em DRG's clínicos quanto cirúrgicos, com desperdício de 71,5 admissões clínicas e 228,7 cirúrgicas no período analisado. A expectativa era de uma permanência média realizada por DRG clínico e cirúrgico de 3,7 e 1,4 dias, respectivamente. No entanto, o que se observou foi uma permanência média realizada de 4,1 dias para internações clínicas e de 1,6 para cirúrgicas.

A taxa de readmissão em 30 dias foi de 3,19\%, com DRG's clínicos e cirúrgicos apresentando proporção semelhante de reinternações. Dados norte-americanos, entretanto, mostram que a rehospitalização precoce é um evento ainda mais frequente, que se relaciona a falhas no seguimento pós-alta e desencadeia aumento de custos e de taxas de morbimortalidade. Corrobora tais achados o fato de 19,6\% dos beneficiários do Medicare terem sido reinternados em até 30 dias entre outubro de 2003 e dezembro de 2004, o que representou um gasto de 17,4 bilhões de dólares em 2004 (JENCKS et al., 2009). 
No Brasil, segundo Dias (2015), no ano de 2011 na cidade de Ribeirão Preto, a taxa de readmissão de 38.145 internações em 11 hospitais, foi de 14,2\%, com variação de 2,1 a 31,7\% entre os hospitais. As readmissões foram mais prevalentes em hospitais com mais de 100 leitos, de alta complexidade e em internações do Sistema Único de Saúde. Os pacientes mais frequentes foram adultos, de ambos os sexos, que apresentaram no mínimo uma comorbidade. Para hospitais participantes de programas de qualidade a taxa de readmissão foi menor, considerando-se a complexidade assistencial e o porte.

Quanto à codificação no hospital em estudo, os DRG's cirúrgicos representaram 69,78\% das altas codificadas, com maior complexidade assistencial em relação aos DRG's clínicos no mesmo período (case mix cirúrgico e clínico de 1,4433 e 0,9879, respectivamente).

A classificação DRG é organizada em 25 categorias diagnósticas maiores (CDM), que são definidas pelo sistema orgânico acometido ou pela etiologia da doença. Cada CDM possui um componente cirúrgico e outro clínico. Todo DRG possui um peso relativo, assim como uma média de permanência aritmética e geométrica. A expressão case mix refere-se à média dos pesos relativos dos DRG's dos pacientes internados em um hospital. Assim, o case mix possibilita a avaliação do nível de morbidade de uma população específica, bem como permite comparar os níveis de morbidade entre diferentes instituições de saúde. Baseando-se no grau de morbidade e sua consequente utilização de recursos, o Medicare estabeleceu o primeiro modelo de pagamento baseado no peso relativo de cada DRG (AIELLO; RODDY, 2017).

No hospital estudado, os DRG's clínicos foram responsáveis por 50,80\% do tempo de permanência realizado e por 79,55\% da proporção de óbitos no período analisado. Assim, muito embora os DRG's clínicos tenham sido menos frequentes e menos complexos, proporcionalmente aos casos cirúrgicos, houve maior consumo de diárias hospitalares e maior taxa de mortalidade nessa população.

A presença de doenças crônicas está associada a maiores taxas de readmissão hospitalar em todos os grupos etários e, portanto, devem ser sempre valorizadas como fator de risco para reinternações precoces (BERRY et al., 2018). Jayakody et al. (2016), em revisão sistemática, também destacaram que doenças crônicas, sobretudo as cardiovasculares, respiratórias e o diabetes mellitus, estão associadas ao aumento das taxas de readmissão em 30 dias. Propuseram-se, então, a avaliar o papel do acompanhamento pós-alta via telefone na redução das readmissões. O seguimento via telefone foi definido como a ligação de um profissional de saúde para o paciente em pós-alta recente com objetivos de: educação, manejo de sintomas, prescrição de medicamentos, reconhecimento precoce de complicações e encaminhamento para 
especialistas ou retorno ao hospital quando necessário. Os 10 artigos incluídos na revisão foram inconclusivos em associar a redução de readmissões ao seguimento via telefone, seja de forma isolada, associado à telemedicina ou a outras intervenções pós-alta. No entanto, os autores reforçaram que aparentemente a integração do acompanhamento telefônico a medidas pré-alta pode ser uma estratégia promissora, muito embora estudos de melhor qualidade devam ser realizados para avaliar tal associação.

Vale destacar ainda que, no hospital em análise, aproximadamente um terço das altas codificadas foram de pacientes com 60 ou mais anos (31,37\% da amostra), população geralmente acometida por uma ou mais comorbidades. Segundo Blakey et al. (2017), as reinternações em idosos não geram apenas risco de piores desfechos clínicos, mas também pioram a percepção do paciente sobre seu tratamento. Os idosos percebem as readmissões como uma experiência negativa e, muitas vezes, sentem que sua saúde (física e emocional) não foi adequadamente tratada pelos profissionais de saúde.

Programações inadequadas de alta estão associadas ao aumento de risco de condições adquiridas e de readmissões não desejadas na população idosa (HESTEVIK et al., 2019; NEITERMAN et al., 2015; RENNKE et al., 2015; MESTEIG et al., 2010). Nesse contexto, vários estudos têm sido realizados para determinar estratégias mais adequadas de alta segura nessa população.

Mansah et al. (2009), em revisão sistemática, observaram as seguintes estratégias e intervenções com impacto positivo na redução de condições adquiridas e no estabelecimento de protocolos de alta segura em pacientes com idade igual ou maior que 65 anos:

- estratégias de comunicação estruturada melhoraram desfechos de pacientes idosos durante o processo de transição hospital-domicílio;

- intervenções conduzidas por enfermeiros e por equipe multidisciplinar foram efetivas em reduzir readmissões em período de 1 a 9 meses;

- intervenções conduzidas por farmacêuticos e equipe multidisciplinar reduziram a frequência de utilização de serviços hospitalares, tais como visitas ao setor de emergência, clínicas de reabilitação e hospitalizações de longa permanência;

- intervenções conduzidas por farmacêuticos foram eficazes em melhorar a qualidade da prescrição médica. Houve também redução significativa de não-aderência em pacientes que necessitavam ingerir 4 ou mais medicações em 3 meses; 
- intervenções de enfermagem foram efetivas em aumentar a qualidade de vida dos pacientes;

- intervenções de enfermagem e da equipe multidisciplinar reduziram custos hospitalares.

Hestevik et. al. (2019), em metanálise composta por 13 artigos e um total de 195 pacientes com idade maior ou igual a 65 anos, destacaram que, durante o processo de alta hospitalar, os pacientes geralmente sentem falta de engajamento dos profissionais de saúde em questionar, discutir e fornecer informações adequadas. Sugerem que, uma vez que a tendência atual é de hospitalização precoce, as seguintes estratégias de "cuidado continuado" devem ser adotadas para tornar o processo de alta mais suave para os idosos:

- fornecer aos idosos informações sobre sua (s) doença (s), medicações, reabilitação e aspectos psicossociais da sua reabilitação;

- incluir no processo de alta hospitalar o atendimento também às necessidades pós-alta dos pacientes, tais como: dieta adequada, equipamentos especiais, necessidade de transporte para acompanhamento pós-alta e cuidados gerais em domicílio. Tais informações devem ser comunicadas para o próximo nível de cuidado do paciente;

- adaptar informações verbais e escritas para o melhor entendimento do paciente. Os profissionais de saúde devem confirmar com os pacientes se realmente entenderam as informações fornecidas;

- envolver e estimular ao paciente a participar do processo de tomada de decisões;

- desenvolver iniciativas para reduzir o sentimento de solidão e isolamento de social pósalta;

- melhorar a comunicação entre provedores de saúde, profissionais, pacientes e seus cuidadores.

Em outra metanálise, que analisou 30 estudos randomizados e controlados, Facchinetti et al. (2020), observaram que medidas de "cuidado continuado" foram efetivas na redução de reinternações em 30 dias especificamente na população idosa. Segundo os autores, esse foi o primeiro estudo a mostrar a associação entre cuidados continuados e redução de readmissões precoces em idosos. Os dados sobre readmissões a longo prazo foram, no entanto, inconclusivos. As medidas de "cuidado continuado", por definição, englobavam três 
dimensões: (1) relacional (associada a força da relação paciente-profissionais de saúde, o que incluiu nível de comunicação, conforto e confiança); (2) informacional (relacionada à disponibilidade de dados documentados em prontuário e adequada compreensão das informações pelos vários profissionais de saúde que atendiam o paciente) e (3) de manejo (ligada à estratégia de cuidados com foco nas necessidades do paciente, que podiam mudar continuamente e deviam ser rapidamente tratadas pelos profissionais que o assistiam). A presença de qualquer uma das dimensões foi associada a redução de readmissões em 30 dias, mas maior efetividade foi observada quando as medidas de cuidados englobaram as três dimensões.

No hospital estudado, pensando-se em reduzir o consumo de diárias desnecessárias, mas sem aumentar as taxas de readmissão em 30 dias, propõe-se o estabelecimento de protocolos de alta segura para todos os pacientes internados, de qualquer de faixa etária, quer seja por DRG's clínicos ou cirúrgicos. O cuidado centrado no paciente, que garante uma melhor experiência com seu tratamento, além de reduzir desperdícios com reinternações não planejadas, deve ser sempre uma prioridade.

Para pacientes com internações clínicas e internações cirúrgicas de emergência, são propostas as seguintes etapas como estratégia de alta segura:

- planejamento de alta: pensar na alta antes mesmo da sua definição e precocemente durante a internação. Avaliar as necessidades de paciente e cuidadores, com auxílio de equipe multidisciplinar (assistentes sociais, psicólogos, fisioterapeutas, fonoaudiólogos e enfermeiros). Atenção especial para pacientes com necessidades de cuidados domiciliares (serviço de home care) - iniciar contato precoce com a fonte financiadora (operadora de saúde);

- educação e preparação de pacientes e cuidadores. Pacientes devem ser orientados sobre autocuidados, receber informações sobre sua doença/tratamento, de forma a sentir-se seguros durante a transição hospital-domicílio e estimulados a aderir ao tratamento. Cuidadores/familiares devem ser orientados e treinados a atender as necessidades dos pacientes em domicílio, tais como: administração de medicamentos, troca de curativos, orientações sobre condições de saúde que necessitam avaliação do paciente em serviços de pronto-atendimento ou em ambulatório;

- definição da forma de seguimento pós-alta (via telefone ou através de agendamento de consulta ambulatorial). Pacientes com baixo risco de reinternação (menores de 
60 anos de idade, sem comorbidades, em pós-operatorio de cirurgias eletivas de pequeno porte, com internações curtas ou sem condições adquiridas hospitalares) devem ser acompanhados por via telefônica. Pacientes com maior risco de reinternação (maiores de 60 anos, portadores de doenças crônicas de qualquer idade, em pós-operatório de cirurgias eletivas de grande porte, com internações prolongadas ou com condições adquiridas durante a internação) devem receber alta com agendamento de consulta ambulatorial (quer seja em consultório do Cirurgião ou Clínico assistente, quer seja no ambulatório da operadora de saúde).

Para pacientes internados para cirurgias eletivas, propõe-se a elaboração de protocolos de ERAS para as principais cirurgias eletivas realizadas no hospital.

Os programas de ERAS foram inicialmente utilizados em cirurgias colorretais eletivas, mas, na prática, todas as especialidades têm sido incentivas a desenvolver e aplicar seus próprios protocolos (NHS, 2020). Esses protocolos envolvem 3 etapas (pré, intra e pósoperatória) que visam preservar as funções fisiológicas do paciente e reduzir o estresse cirúrgico, com consequente redução do tempo de recuperação após a cirurgia (NICHOLSON et al., 2014).

Segundo Blumenthal (2019) e Lau et al. (2016), os seguintes componentes devem ser priorizados em cada uma das etapas de um protocolo de ERAS:

- Pré-operatória: fornecimento de informações e aconselhamento médico minucioso pré-cirurgia; diretrizes de jejum e ingestão de carboidratos; eliminação de preparações intestinais mecânicas; profilaxias antimicrobiana e para tromboembolismo venoso;

- Intra-operatória: emprego de analgésicos multimodais não-opióides e antieméticos; preferência para anestesia local e regional; manutenção de normotermia e euvolemia;

- Pós-operatória: mobilização e deambulação precoces; prevenção de náuseas e vômitos; nutrição oral precoce; uso de analgésicos multimodais não-opióides e remoção precoce de tubos, drenos, cateteres e sondas.

Sibbern et al. (2016), em revisão sistemática, investigaram, de forma qualitativa, a experiência de pacientes com o emprego do ERAS. Os autores observaram que, para aumentar a sensação de segurança do paciente e a sua aderência aos protocolos, foram fundamentais: (1) engajamento de profissionais de saúde que assistiram os pacientes e (2) fornecimento adequado 
de informações sobre o procedimento cirúrgico e sua recuperação no pré e pós-operatório. Nesse trabalho, foram destacados ainda a relevância do seguimento pós-alta (seja por telefone ou visitas médicas) e a importância do envolvimento e fornecimento de informações para familiares/cuidadores na percepção de valor entregue ao paciente.

Varandhan et al. (2010) conduziram a primeira metanálise a avaliar as diferenças de desfechos entre pacientes submetidos a esses protocolos e aqueles com manejo cirúrgico convencional. Os autores observaram uma redução significativa de tempo de internação hospitalar com a utilização de protocolos de ERAS em cirurgias colorretais eletivas abertas, mas não houve diferenças nas taxas de mortalidade e de readmissão entre os dois grupos. Uma limitação desse trabalho foi a pequena quantidade de estudos incluídos - apenas seis.

Nicholson et al. (2014) observaram dados semelhantes, mas em uma revisão sistemática composta por 38 artigos, com um total de 5.099 pacientes e outras especialidades cirúrgicas incluídas. Houve redução significativa de tempo de internação e de incidência de complicações dentro de 30 dias nos pacientes que participaram de protocolos de ERAS em comparação com pacientes submetidos ao manejo cirúrgico convencional. Não houve diferenças nas taxas de mortalidade e de readmissões entre os grupos. Na subdivisão das complicações em subgrupos, também não houve redução de complicações maiores, definidas como: trombose venosa profunda, tromboembolismo pulmonar, infarto agudo do miocárdio e necessidade de reintervenção cirúrgica. Observou-se ainda que o impacto do emprego de protocolos de ERAS foi semelhante entre as várias especialidades cirúrgicas.

Dois estudos mais recentes mostraram também vários benefícios da utilização dos programas de ERAS nas várias especialidades cirúrgicas. Lau et al. (2016), em metanálise composta por 42 estudos e um total de 5.241 pacientes (2.595 no grupo ERAS e 2.646 no grupo de manejo convencional), observaram que os protocolos de ERAS se associaram à redução de tempo de internação, de complicações totais e custos totais. Além disso, notou-se retorno mais precoce da função do trato gastrointestinal e não foram observados impactos nas taxas de mortalidade e nas readmissões em 30 dias. A exceção foram os pacientes submetidos à cirurgia gastrointestinal, subgrupo de pacientes no qual houve aumento das readmissões em 30 dias.

Visioni et al. (2018) conduziram revisão sistemática e metanálise constituída por 39 estudos e 6.511 patientes (3.456 no grupo ERAS e 3055 no grupo controle), sendo incluídos estudos com cirurgias abdominais não colorretais. Observou-se uma redução de tempo de internação e de custos no grupo de pacientes dos programas ERAS, sem aumento, no entanto, de complicações e de readmissões em 30 dias nesse grupo. Os autores concluíram que os 
protocolos de ERAS são seguros, eficazes em reduzir custos e tempo de internação, sendo benéficos para pacientes cirúrgicos de múltiplas especialidades e para o sistema de saúde. Acreditam também que os protocolos de ERAS se tornarão o padrão em cirurgias abdominais e serão o principal componente para melhoria de qualidade e entrega de valor em pacientes cirúrgicos.

\section{CONSIDERAÇÕES FINAIS}

O uso da ferramenta DRG pode auxiliar, através da análise de seus relatórios assistenciais, a elaboração de protocolos de alta segura que visam melhorar a qualidade assistencial e a satisfação dos pacientes. Acredita-se que consequentemente ocorrerá, também, a redução de desperdícios e de custos assistenciais, com aumento de viabilidade financeira e competitividade da instituição.

Com base na avaliação de relatórios gerados pelo sistema DRG, propõe-se, para o hospital em estudo, as seguintes estratégias de alta segura:

1. Para internações clínicas e internações cirúrgicas de emergência, são propostas as seguintes etapas: (A) planejamento de alta; (B) educação e preparação de paciente e cuidadores e (C) definição da forma de seguimento pós-alta (via telefone ou através de agendamento de consulta ambulatorial conforme o risco de readmissão em 30 dias);

2. Para para cirurgias eletivas, sugere-se a elaboração de protocolos de ERAS para as principais cirurgias eletivas realizadas no hospital.

\section{Referências}

AIELLO, Francesco A.; RODDY, Sean P.. Inpatient coding and the diagnosis-related group. Journal Of Vascular Surgery, [S.L.], v. 66, n. 5, p. 1621-1623, nov. 2017. Elsevier BV.

BARNES, S.; HAMROCK, E.; TOERPER, M.; SIDDIQUI, S.; LEVIN, S. Real-time prediction of inpatient length of stay for discharge prioritization. JAMIA, 2016; 23(e1):e2e10. https://academic.oup.com/jamia/article/23/e1/e2/2379761.

BERRY, Jay G; GAY, James C; MADDOX, Karen Joynt; A COLEMAN, Eric; BUCHOLZ, Emily M; O’NEILL, Margaret R; BLAINE, Kevin; HALL, Matthew. Age trends in 30 day hospital readmissions: US national retrospective analysis. Bmj, [S.L.], p. k497-k505, 27 fev. 2018. BMJ. 
BLAKEY, Emma Pascale; JACKSON, Debra; WALTHALL, Helen; AVEYARD, Helen. What is the experience of being readmitted to hospital for people 65 years and over? A review of the literature. Contemporary Nurse, [S.L.], v. 53, n. 6, p. 698-712, 2 nov. 2017. Informa UK Limited.

BLUMENTHAL, Rebecca N.. ERAS: Roteiro para uma jornada segura no perioperatório. Boletim da Anesthesia Patient Safety Foundacion 2019;34:22-24.https://www.apsf.org/wpcontent/uploads/newsletters/2019/0201-pt-br/APSF0201-PT-BR.pdf.

CALDERÓN, Venancio García et al. The impact of improving the quality of coding in the utilities of Diagnosis Related Groups system in a private healthcare institution. 14-year experience. International Journal of Medical Informatics, [s.1.], v. 129, p.248-252, set. 2019. Elsevier BV.

CORTÉS, Ariel Emilio et al. Cultura innovadora y grupos relacionados de diagnóstico en un hospital de alta complejidad, Colombia. Revista de Salud Pública, [s.1.], v. 18, n. 2, p.251262, 2016. Universidad Nacional de Colombia.

COUTO, R. C.; Pedrosa, T.M.G.; ROBERTO, B.A.D.; DAIBERT, P.B.. DRG Brasil: transformando o sistema de saúde brasileiro e a vida das pessoas. Belo Horizonte: MG, 2018. Publicação digital (ePub).

DIAS, Bruna Moreno. Readmissão hospitalar como indicador de qualidade. Dissertação de Mestrado, [S.L.], 18 set. 2015. Universidade de São Paulo, Agência USP de Gestão da Informação Acadêmica (AGUIA).

FACCHINETTI, Gabriella; D’ANGELO, Daniela; PIREDDA, Michela; PETITTI, Tommasangelo; MATARESE, Maria; OLIVETI, Alice; MARINIS, Maria Grazia de. Continuity of care interventions for preventing hospital readmission of older people with chronic diseases: a meta-analysis. International Journal Of Nursing Studies, [S.L.], v. 101, p. 103396-103406, jan. 2020. Elsevier BV.

HESTEVIK, Christine Hillestad; MOLIN, Marianne; DEBESAY, Jonas; BERGLAND, Astrid; BYE, Asta. Older persons' experiences of adapting to daily life at home after hospital discharge: a qualitative metasummary. Bmc Health Services Research, [S.L.], v. 19, n. 1, p. 224-237, 11 abr. 2019. Springer Science and Business Media LLC.

JAYAKODY, Amanda; BRYANT, Jamie; CAREY, Mariko; HOBDEN, Breanne; DODD, Natalie; SANSON-FISHER, Robert. Effectiveness of interventions utilising telephone follow up in reducing hospital readmission within 30 days for individuals with chronic disease: a systematic review. BMC Health Services Research, vol. 16, n. 1, p. 403-411, 18 aug. 2016.

JENCKS, Stephen F.; WILLIAMS, Mark V.; COLEMAN, Eric A.. Rehospitalizations among Patients in the Medicare Fee-for-Service Program. New England Journal Of Medicine, [S.L.], v. 360, n. 14, p. 1418-1428, 2 abr. 2009. Massachusetts Medical Society.

KEHLET, Henrik; WILMORE, Douglas W.. Evidence-Based Surgical Care and the Evolution of Fast-Track Surgery. Annals Of Surgery, [S.L.], v. 248, n. 2, p. 189-198, ago. 2008. Ovid Technologies (Wolters Kluwer Health). 
LAU, Christine S. M.; CHAMBERLAIN, Ronald S.. Enhanced Recovery After Surgery Programs Improve Patient Outcomes and Recovery: a meta-analysis. World Journal Of Surgery, [S.L.], v. 41, n. 4, p. 899-913, 7 nov. 2016. Springer Science and Business Media LLC.

LEPPIN, Aaron L.; GIONFRIDDO, Michael R.; KESSLER, Maya; BRITO, Juan Pablo; MAIR, Frances S.; GALLACHER, Katie; WANG, Zhen; ERWIN, Patricia J.; SYLVESTER, Tanya; BOEHMER, Kasey. Preventing 30-Day Hospital Readmissions. Jama Internal Medicine, [S.L.], v. 174, n. 7, p. 1095-1107, 1 jul. 2014. American Medical Association (AMA).

MANSAH, Martha; FERNANDEZ, Ritin; GRIFFITHS, Rhonda; CHANG, Esther. Effectiveness of strategies to promote safe transition of elderly people across care settings. Jbi Library Of Systematic Reviews, [S.L.], v. 7, n. 24, p. 1036-1090, 2009. Ovid Technologies (Wolters Kluwer Health).

MENG, Zhaolin; HUI, Wen; CAI, Yuanyi; LIU, Jiazhou; WU, Huazhang. The effects of DRGs-based payment compared with cost-based payment on inpatient healthcare utilization: a systematic review and meta-analysis. Health Policy, [S.L.], v. 124, n. 4, p. 359-367, abr. 2020. Elsevier BV.

MESTEIG, Marianne; HELBOSTAD, Jorunn L; SLETVOLD, Olav; RØSSTAD, Tove; SALTVEDT, Ingvild. Unwanted incidents during transition of geriatric patients from hospital to home: a prospective observational study. Bmc Health Services Research, [S.L.], v. 10, n. 1, p. 1-9, 4 jan. 2010. Springer Science and Business Media LLC.

NEITERMAN, Elena; WODCHIS, Walter P.; BOURGEAULT, Ivy Lynn. Experiences of Older Adults in Transition from Hospital to Community. Canadian Journal On Aging / La Revue Canadienne Du Vieillissement, [S.L.], v. 34, n. 1, p. 90-99, 30 dez. 2014. Cambridge University Press (CUP). http://dx.doi.org/10.1017/s0714980814000518.

NHS Institure for Innovation and Improvent. Enhance Recovery Programme. https://www.uhs.nhs.uk/OurServices/Surgery/Enhancedrecoveryprogramme/Enhancedrecover yprogramme-aftersurgery.aspx. Acesso 26 nov. 2020.

NICHOLSON, A.; LOWE, M. C.; PARKER, J.; LEWIS, S. R.; ALDERSON, P.; SMITH, A. F.. Systematic review and meta-analysis of enhanced recovery programmes in surgical patients. British Journal Of Surgery, [S.L.], v. 101, n. 3, p. 172-188, 27 jan. 2014. Wiley.

NORONHA, Marina F.; VERAS, Cláudia T.; LEITE, Iuri C.; MARTINS, Monica S.; BRAGA NETO, Francisco; SILVER, Lynn. O desenvolvimento dos "Diagnosis Related Groups" - DRGs. Metodologia de classificação de pacientes internados. Revista de Saúde Pública, [S.L.], v. 25, n. 3, p. 198-208, jun. 1991. FapUNIFESP (SciELO).

PORTER, Michael E.. What Is Value in Health Care? New England Journal Of Medicine, [S.L.], v. 363, n. 26, p. 2477-2481, 23 dez. 2010. Massachusetts Medical Society.

PORTER, M. E.; LEE, T. H. The Strategy That Will Fix Healthcare. Harvard Business Review, v. 1277, n. October, p. 1-19, 2013. 
PORTER, M. E.; TEISBERG, E. O. Redefining health care: Creating Value-Based

Competition on Results. 1. ed. Boston: Harvard Business School Press, 2006.

RENNKE, Stephanie; RANJI, Sumant R.. Transitional Care Strategies From Hospital to Home. The Neurohospitalist, [S.L.], v. 5, n. 1, p. 35-42, 10 jul. 2014. SAGE Publications.

RIMLER, Simcha B.; GALE, Brian D.; REEDE, Deborah L.. Diagnosis-related Groups and Hospital Inpatient Federal Reimbursement. Radiographics, [S.L.], v. 35, n. 6, p. 1825-1834, out. 2015. Radiological Society of North America (RSNA).

http://dx.doi.org/10.1148/rg.2015150043.

SANTOS, Izabelle Queiroz dos et al. Comparação De Estratégias Para Detecção De Condições Adquiridas Em Hospital Público Segundo O Sistema De Grupos Diagnósticos Relacionados (DRG). Revista de Gestão em Sistemas de Saúde, [s.1.], v. 7, n. 1, p.44-55, 1 abr. 2018. University Nove de Julho.

SIBBERN, Tonje; SELLEVOLD, Vibeke Bull; A STEINDAL, Simen; DALE, Craig; WATT-WATSON, Judy; DIHLE, Alfhild. Patients' experiences of enhanced recovery after surgery: a systematic review of qualitative studies. Journal Of Clinical Nursing, [S.L.], v. 26, n. 9-10, p. 1172-1188, 9 fev. 2017. Wiley.

SMITH, M.D.; McCALL, J.; PLANK, L.; HERBINSON, G.P.; SOOP, M.; NYGREN, J. Preoperative carbohydrate treatment for enhancing recovery after selective surgery.

Cochrane Database of Systematic Reviews 2014, Issue8. Art. No.: CD009161.

VISIONI, Anthony; SHAH, Rupen; GABRIEL, Emmanuel; ATTWOOD, Kristopher; KUKAR, Moshim; NURKIN, Steven. Enhanced Recovery After Surgery for Noncolorectal Surgery? Annals Of Surgery, [S.L.], v. 267, n. 1, p. 57-65, jan. 2018. Ovid Technologies (Wolters Kluwer Health).

VARADHAN, Krishna K.; NEAL, Keith R.; DEJONG, Cornelius H.C.; FEARON, Kenneth C.H.; LJUNGQVIST, Olle; LOBO, Dileep N.. The enhanced recovery after surgery (ERAS) pathway for patients undergoing major elective open colorectal surgery: a meta-analysis of randomized controlled trials. Clinical Nutrition, [S.L.], v. 29, n. 4, p. 434-440, ago. 2010. Elsevier BV. 